\title{
Palliative neurosurgies for recurrent seizures in a child with single ventricle physiology at different stages of cardiac palliation: an anaesthetic and neurosurgical challenge
}

Kremer Y., Sanchez Torres C., Momeni M., Docquier M.-A.

Cliniques Universitaires Saint Luc; Université Catholique de Louvain, Dept of Anaesthesiology, Brussels, Belgium

\section{Background}

Anaesthetic management of paediatric patients with single ventricle (SV) physiology is challenging. We report two epileptic neurosurgical procedures in a boy with SV physiology at two different stages of cardiac palliation.

\section{Case report}

A 18-months-old boy with SV physiology suffering from stroke after bidirectional superior cavopulmonary connection (CPC) necessitated a callosotomy (palliative neurosurgery) for refractory seizures. A multidisciplinary discussion was planned to cope with his particular cardiac physiology (high superior caval venous pressures and the existence of intracardiac shunts) and the specific neurosurgical requirements being the necessity to improve cerebral relaxation. Blood pressure was measured invasively. A central venous catheter (CVC) was placed in the right internal jugular vein to measure the pulmonary pressures. Another CVC placed in the femoral vein measured the single atrium pressure. Cerebral NIRS was used. Intraoperatively, mannitol was avoided (to reduce the risk of paradoxal air embolism), normocapnia was maintained and an intraventricular catheter (IVC) was placed to reduce intracranial volume. The surgery and postoperative course were uneventful (Surgery 1).

The boy was scheduled 4 years later for a hemispherotomy because of recurrent untractable seizures. Meanwhile, he had undergone total CPC (Fontan) with an extracardiac conduit and a fenestration which had been closed percutaneously before his neurosurgery. Intraoperatively there were no episodes of hypovolaemia, hypotension or hypoxemia. The CVC was inserted in the femoral vein which presented the pulmonary artery pressure. Mannitol was avoided to reduce the risk of relative hypovolaemia. An IVC was also placed to optimize surgical conditions. The surgery and postoperative course were uneventful (Surgery 2)
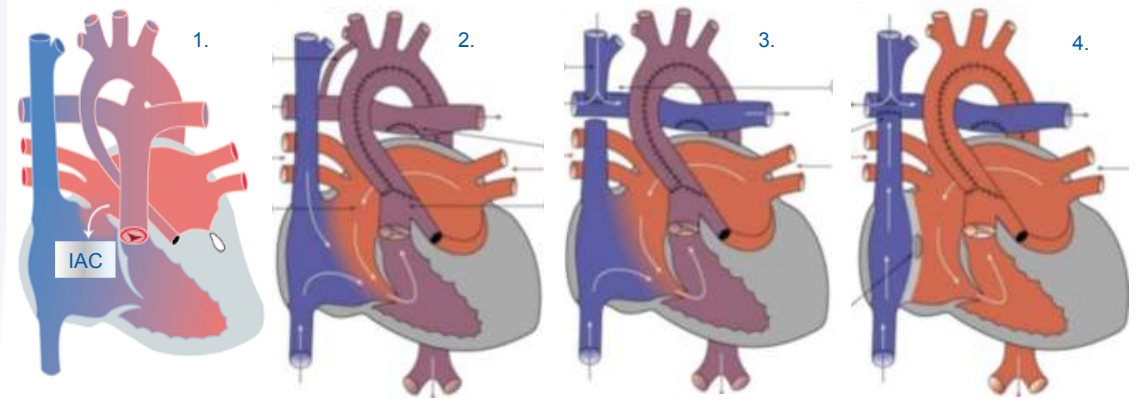

Different stages of cardiac palliation:

1. SV physiology at birth

2. Blalock-Taussig shunt

3. Bidirectional superior CPC (Glenn)

4. Total CPC/Fontan correction

Modified from Barry A, Transcatheter superior cavopulmonary anastomosis: interresting technique, limited applicability. J Am Coll Cardiolog. 70; 2017

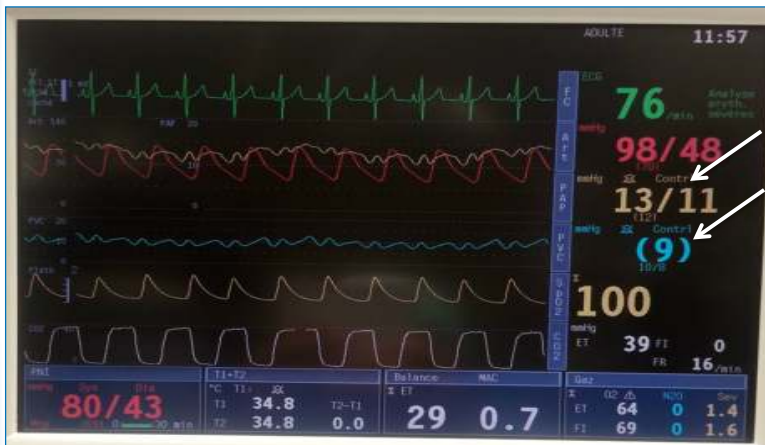

Surgery 1: A CVC was placed in the right internal jugular vein and measured the pulmonary pressures. Another CVC placed in the femoral vein measured the single atrium pressure.

Surgery 2: The CVC was inserted in the femoral vein which presented the pulmonary artery pressure.

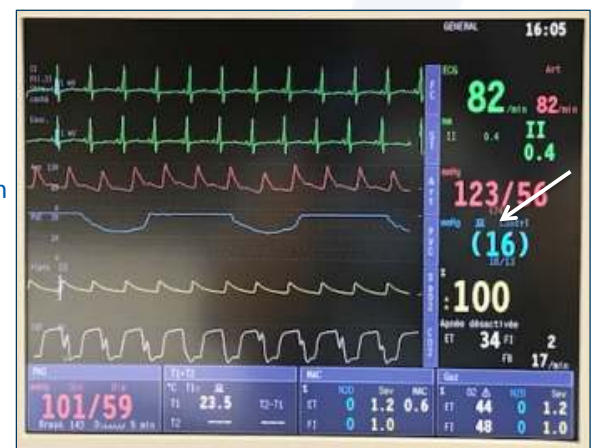

Discussion

Anaesthetic management of children with SV physiology presenting for neurosurgery is challenging but radically different at the different stages of cardiac palliation. The anaesthesiologist has to be aware of the impact of preload modifications and ventilation techniques on hemodynamic parameters and cerebral relaxation to find the most balanced anaesthetic approach.

\section{References}

1. Flack S et al. Cerebral Hemispherectomy in Infants and Young Children. Paediatr Anaesth 2008; 18(10):967-73. 2. Scallan MJ. Brain Injury in Children with Congenital Heart Disease. Paediatr Anaesth 2003; 13(4):284-93.

\section{Learning points}

Children with SV physiology nowadays undergo non-cardiac surgery. Neurosurgery remains challenging in these sick children because of specific surgical conditions. Multidisciplinary discussion and understanding of cardiac physiology at different stages is mandatory. 